\title{
Social demographics, snack consumption, vending machine use and oral health
}

\author{
The effect of social demographic factors, snack consumption and vending machine use on oral health of children \\ living in London M. Maliderou, S. Reeves and C. Noble Br Dent J 2006; 201: 441-444
}

\begin{abstract}
Objective
To investigate the effect of socio-economic status, sugar, snack consumption and vending machine use on the prevalence and severity of caries (DMF) in children.

Design

An observational study was carried out in a dental practice in inner city London. Sixty children were asked to complete a questionnaire and a three day food and drink diary. After a dental examination the number of decayed (D), missing (M) or filled (F) teeth provided a DMF score. Anova and Pearsons correlations were used to analyse the data statistically. Results

Children from social groups I and II consumed significantly less $(\mathrm{P}<$ 0.05) sugar, confectionery, crisps and used a vending machine less often than children from other social groups. Children from Social groups I, II and III had significantly lower DMF scores. The average DMF from social group I children was $0.5 \pm 0.6$, whilst group IV children had the greatest incidence and a DMF of $4.6 \pm 0.8$. Significant correlations were identified between DMF and sugar, confectionery and crisp consumption and vending machine use, and a negative correlation between DMF and vegetable consumption.

Conclusions

Socio-economic status and access to vending machines were found to have a significant effect on sugar intakes, foods choices, and dental health. The removal of vending machines from schools or at least installing 'healthy' vending machines is recommended. Health promotion programmes that account for social groups and snacking habits that are cost effective are required.
\end{abstract}

\section{IN BRIEF}

- Diet, choice of snack food and socio-economic status were shown to affect dental health.

- Level of obesity was not seen to influence DMF score or oral health.

- Vending machines in schools can be hazardous to dental health and should be removed or replaced with 'healthy' vending machines.

- Health promotion should focus on the role of snacking and vending machines in schools.

\section{COMMENT}

Since 1954 and the infamous Vipeholm Study, ${ }^{1}$ the effect of diet and the frequency of sugary snack consumption on the prevalence and severity of caries has been recognised and accepted. That the prevalence and severity of dental caries in children might be related to social class and the use of vending machines in schools is a logical corollary.

In the wake of Jamie Oliver's recent pioneering publicity for healthier school meals for kids, this study is timely and emphasises the need for an evidence-based approach to school attitudes to all food and drink consumed on their premises and the importance of ease of access to various food types in influencing children's dietary habits.

Hence the positive correlation between the use in schools of vending machines, which are usually associated with the sale of sugar and fatty snacks, and a raised DMF score in children. The complementary role of school in affecting and possibly countering the effect of social class on dietary intake makes an interesting hypothesis!

That a large proportion of the group of 60 children studied were overweight or obese and the existence of a strong negative correlation between vegetable consumption and DMF (probably because those children who consumed the greatest numbers of portions of fruit and vegetables per day also consumed the least sugar and confectionery items) suggests the holistic relevance of diet to general as well as oral wellbeing.

The reliability and validity of information obtained from 'self assessed' food and drink diaries is understandably questionable. However the use of a questionnaire to obtain further specific information in this study acted as a useful cross reference tool to highlight any inaccuracies. The potential for conditioning children's dietary habits is implicit throughout this study.

The importance of targeting high risk groups with feasible, affordable and practical dietary and oral hygiene advice is self evident but the results of this study also have far reaching implications for future public oral health strategies, for example the re-evaluation of the use of vending machines in schools and the positive effect of healthy dietary alternatives (fruit and vegetables) in reducing the consumption of sugar and sugary snacks.

K. Barnard, Consultant in Paediatric Dentistry, Chelsea and Westminster Hospital, London

1. Gustaffson B E, Quensel CE, Lanke LS et al. The Vipeholm dental caries study: the effect of different levels of carbohydrate intake on caries activity in 436 individuals for five years. Acta Odontol Scand 1954; 11: 232-365.

Ol:10.1038/sj.bdj.4814123 